\title{
AGENT-BASED PERFORMANCE ASSESSMENT TOOL FOR GENERAL AVIATION OPERATIONS UNDER FREE FLIGHT
}

\author{
Karen A. Harper ${ }^{*}$, Sandeep S. Mulgund ${ }^{\dagger} \&$ Greg L. Zacharias ${ }^{\ddagger}$ \\ Charles River Analytics \\ One Alewife Center \\ Cambridge, MA 02140 \\ James K. Kuchar ${ }^{\S}$ \\ Department of Aeronautics and Astronautics \\ Massachusetts Institute of Technology \\ Cambridge, MA 02139
}

\begin{abstract}
The objective of this research is to design and demonstrate an agent-based modeling and analysis tool for evaluating General Aviation (GA) pilot situation awareness under free flight air traffic management (ATM). A computational tool is developed to assess free flight's potential effect on GA operators, by combining an agent-based representation of the overall pilot/vehicle/ATM system with quantitative modelbased metrics of pilot SA. The model's performance is demonstrated in a set of simulation trials designed to measure the pilot agent's ability to recognize and correctly assess protected zone conflicts in free flight ATM, using information available from a hypothetical cockpit display of traffic information. A set of simulations is presented to examine the effect of sensor accuracy and attention allocation on pilot awareness of protected zone conflict hazards posed by intruder aircraft. The results show that reducing sensor accuracy leads to an increase in overall SA error, and that the pilot agent divides its attention over multiple traffic hazards in proportion to each intruder's hazard potential. This attention-sharing varies dynamically as the conflict situation changes, in a manner that is consistent with intuitive expectations.]
\end{abstract}

\section{INTRODUCTION}

Nascent concepts for free flight air traffic management (ATM) will dramatically change human roles and tasks in the airspace system. Free flight, which has been defined as "a safe and efficient flight operating capability under instrument flight rules (IFR) in which operators have the freedom to select their path and speed in real time" [1], will have profound implications on pilot information requirements, pilot/controller roles

\footnotetext{
${ }^{*}$ Research Engineer. Member AIAA.

${ }^{\dagger}$ Senior Scientist. Senior Member, AIAA.

$\stackrel{+}{*}$ Principal Scientist. Member, AIAA.

$\S$ Assistant Professor. Member, AIAA.

Copyright (C) 1998 by the American Institute of Aeronautics and Astronautics, Inc. All rights reserved.
}

and responsibilities, workload allocation, communication, and decision-making. Such systemic changes will inevitably introduce new human factors challenges and new sources of human error. It has often been the case that human factors issues are normally only addressed very late in development, often too late to facilitate necessary changes [2]. If it is accepted as fact that aviation safety not be compromised in a transition to free flight, human factors issues should be addressed concurrently with the development of free flight technologies and procedures.

Although continuing advances in on-board automation and aiding systems should ameliorate safety concerns in the commercial cockpit, there is considerable concern regarding the impact of free flight on the general aviation (GA) community. This community represents approximately $95 \%$ of all aircraft and $60 \%$ of aircraft operations hours in the continental United States. Thus far, the consequences of free flight on GA have received relatively little attention. The safety of GA operations under free flight will hinge on the adequacy of the equipment needed for free flight and the operating procedures that support it [3]. Of specific importance are the requirements for supporting adequate pilot situation awareness (SA).

Situation awareness, which refers to a pilot's internal model of the world around him at any point in time [4], is the starting point for pilot decision-making, particularly in abnormal or emergency situations [5]). The pilot obtains information to maintain awareness of the flight situation visually (through aircraft windows and from instruments), aurally (from other crewmembers and over radio communications), and through vestibular senses. The information thus obtained forms the basis of the pilot's decisions. Since all of the pilot's information-gathering skills are subject to error, failures in maintaining adequate SA can severely impact flight safety. For example, a recent study of fatal commercial aircraft accidents involving controlled flight into terrain (CFIT) implicates loss of SA as a dominant factor [6]. 
If the search for solutions to problems of human situation awareness and decision-making error is to be efficient, it is important to leverage the insight gained through decades of research in the behavioral sciences [7]. An agent-based approach to assessing the human factors impact of free flight should account for the pilot's fundamental capabilities and limitations in processing and acting upon information, starting with his limited performance in sensory/perceptual processing and data fusion, proceeding to his imperfect strategies for situation assessment and decision-making, and following through to his sometimes less than optimal execution of a range of procedural activities. The approach must be able to represent pilot interaction with a variety of automation concepts, from full-level non-advisory automatic systems, to limited-authority advisors. There should also be provision for growth and modification of the method, as ATM concepts evolve. The approach should account for other on-board design factors that impinge upon cockpit workload such as new navigation aids, communication protocols, etc., and there should exist a means of incorporating these factors in a relatively straightforward fashion.

These capabilities are afforded by an agent-based approach founded on a detailed behavioral representation of the overall pilot/vehicle/ATM system. Such a representation can provide the critical information-based linkage between the external environment (including the ATM system), the vehicle, the cockpit interface, and the pilot. It can serve as a framework for integrating the pilot's knowledge base with his procedural knowledge, to provide insight as to how the pilot assesses situations, makes decisions, executes procedures, and conducts communications. The pilot model employed here is based on the structured formalization in the Crew/System Integration Model (CSIM) [8], which has recently been applied in fighter/attack mission and air superiority modeling efforts [9]. Such a pilot agent model provides a means for locating sources of error at various points in the information processing chain, and at various levels at each location. This explicit representational approach can account for error-free behavior on the part of the pilot, and it can also be used to directly evaluate alternative hypotheses regarding error sources, their effect on nominal information processing by the pilot, and their impact on overall pilot/vehicle performance and flight safety. This agent-based modeling approach will enable ATM technology designers to:

- Use a model-based approach to identify the effects of system design on pilot SA and performance

- Populate air traffic simulation models with "intelligent" air traffic hazards, for evaluation of candidate flight deck technologies using a realistic air traffic model
- Leverage the computational SA technology underlying the agent model to support pilot SA in the cockpit, by having it drive display content on cockpit displays of traffic information

- Support human error modeling and analysis under NASA's Aviation Safety Program [10], which seeks to improve aviation safety five-fold over the next 10 years through technology development for accident prevention, accident mitigation, and development of system-level concepts for improving safety

The primary objective of this work is to design and demonstrate an agent-based modeling and analysis tool for evaluating General Aviation (GA) pilot situation awareness (SA), performance, and error rates as a function of free flight design variables (such as separation criteria, adaptive sector strategy, traffic density limits, alert zone shape and size, etc.).

\section{FUNCTIONAL SYSTEM DESIGN}

\section{Overall System Architecture}

Figure 1 presents the overall architecture of the agent-based model for evaluation of pilot SA and performance under free flight. The system contains the following key components:

- An analytical model of the GA pilot agent, representing the key activities of information processing, situation assessment, and decisionmaking (IP, SA, and DM, respectively)

- A pilot agent SA/performance metric generator, which quantitatively estimates model-based metrics of pilot SA

- $\quad$ The Free Flight ATM Executive, which models the free flight setup (flight rules, separation criteria, etc.), and plays the role of air traffic control (ATC)

As shown, the pilot agent is instantiated $N$ times, to represent $N$ aircraft operating within the simulation (note that a given aircraft may actually contain both a pilot and copilot; this would be a straightforward extension of what is shown here). Each of these aircraft communicates with ATC via simulated voice and datalink, as required. The effect of mixed equipage is handled in a straightforward manner by changing the definition of the information available to each aircraft; new or absent equipment may be represented by adding to or removing from the information set available to a pilot. Information accuracy and bandwidth may be modulated by changing the information set dynamics and noise levels. Varying levels of pilot expertise may be represented by changing underlying model parameters (situation assessment and decision models, control gains, etc.). 


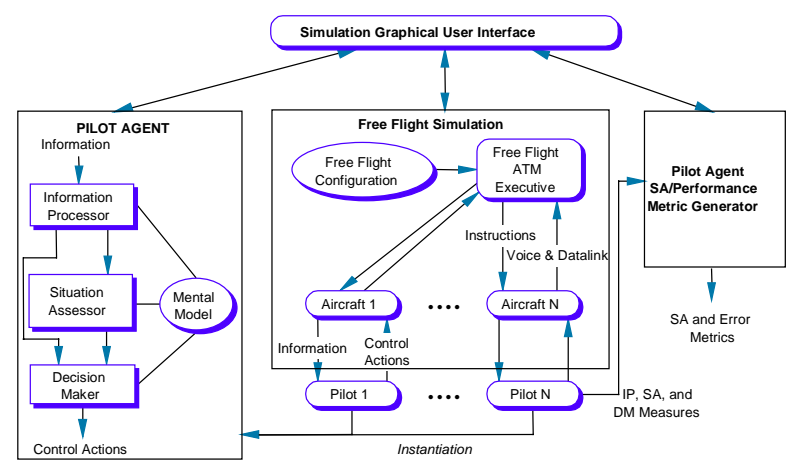

Figure 1: Overall System Architecture

The Free Flight ATM executive models the "ground-based" portion of the free flight simulation. This module includes several components:

- A traffic situation generator

- A conflict detection and resolution module

- An air traffic control/simulation control station

The traffic situation generator provides aircraft flight plans either using scripted plans or through statistical techniques (e.g., desired traffic density, speeds, and potential routes), which in turn are used to define initial conditions and flight plans for each of the aircraft/pilot agent instantiations. An air traffic/simulation control station will also be interfaced with the simulation to incorporate parameters such as separation criteria, assumed response time, adaptive sectors, and conflict resolution algorithms.

\section{Pilot Agent Model Architecture}

Figure 2 illustrates the central position that situation awareness holds in the pilot agent model, and in the studies of many researchers, including Klein [11], Smith \& Sage [12], and Zacharias, Miao, Illgen, Yara \& Siouris [9]. Six basic steps are involved:

1) Monitor the environment: Given the situation status established by an internal mental model of the scenario, a decision maker monitors the environment looking for event cues that confirm or disconfirm the current assessed situation.

2) Determine the need for situation assessment: If the event cues are consistent with the assessed situation, continue monitoring (loop back to step 1 ); if they are not, proceed to the next step.

3) Propagate event cues: Based on the mental model, propagate the newly received event cues to start a new round of situation assessment. The result of this event cue propagation generates a new belief distribution among situations, and can lead to a new assessment of the situation.

4) Anticipate events: Based on the updated situational beliefs, predict situation-related event occurrences. These anticipated or predicted events feed back to guide the decision-maker's event monitoring strategy used in step 1.

5) Assess situation: Determine whether the updated situational beliefs support the confident assessment of a new situation (or situations). If the answer is yes, proceed to step six; otherwise, continue monitoring for new events.

6) Make decision: If a new situation is assessed, a new decision procedure associated with the situation, is called upon, and acted upon to generate new situationally relevant actions.

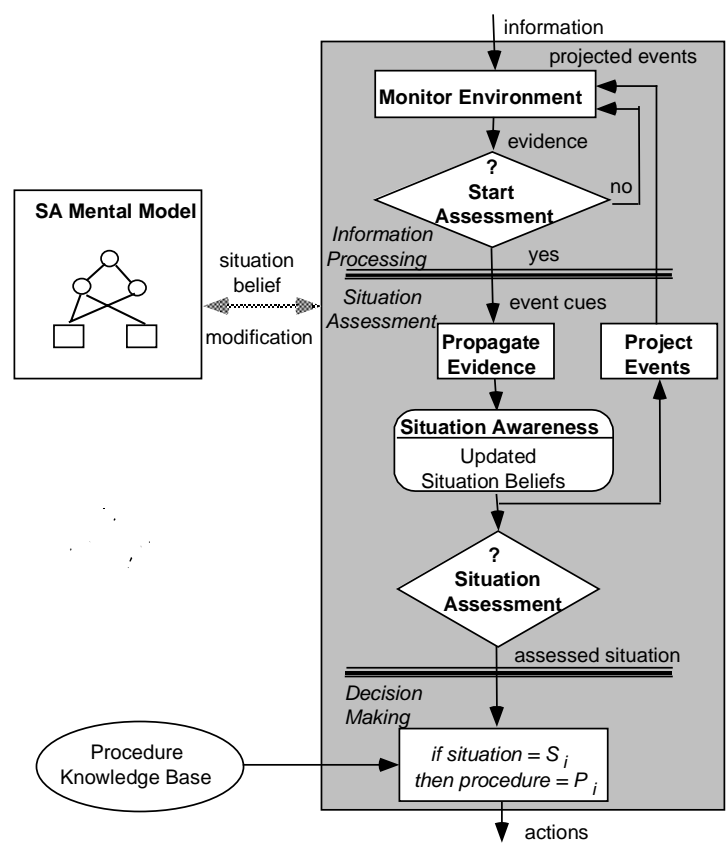

Figure 2: SA-Centered Decision-Making

Notice the difference between this SA centered model and the conventional decision-making model that views the decision maker as "faced with alternatives, and considering the consequences of each alternative in terms of analysis of future states (odds/probabilities) weighed against alternative goals (preferences/utilities)" [11]. In the SA centered model, no utility or alternative is considered; instead, SA becomes the focus of all pilot actions. It not only defines a decision maker's view of his/her environment, but also serves to define his/her information needs and to drive his/her effective experiential (if-then) decision-making.

Figure 3 decomposes this module into its constituent algorithmic components, which are:

- An information processor that processes information generated by the simulation, to yield system states and event cues

- A situation assessor that uses event cues to generate the current assessed situation 
- A decision-maker that selects among alternative procedures to produce control actions, based on the current flight situation and estimated states

This model is founded on the Crew/System Integration Model [9], which is an informationprocessing model of the human operator of a dynamic system. The pilot model employs two key technologies: a belief network (BN) [13] representation of the pilot's SA functions; and an expert system (ES) implementation of the pilot's DM activities. The model is supported by a scenario-dependent mental model that maintains the structure and parameters defining the event/situation relationships, as well as the procedural rules defining the pilot's decision-making strategy [14]. These procedural rules may specify pilot behavior under candidate free flight rules.

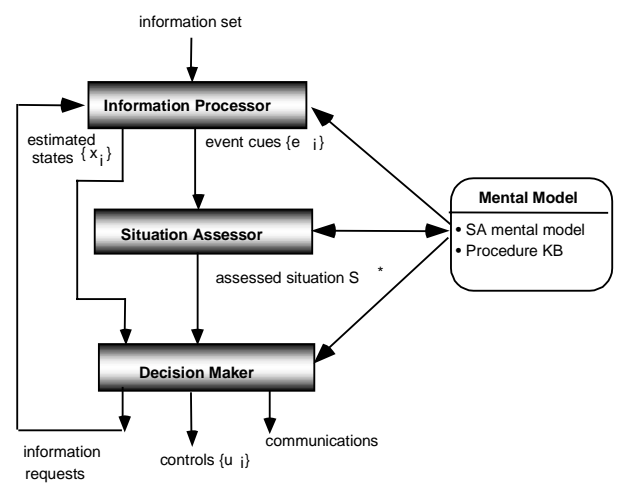

Figure 3: Architecture of Pilot Mental Model

\section{Situation Assessment Model}

The first key component of the system architecture is the current situation assessment module, which uses aircraft information system outputs to generate a highlevel interpretation of the current air traffic situation facing the pilot. The system described here relies on belief networks [13] for reasoning in the presence of uncertainty.

Any robust computational model of situation assessment requires a technology that has: 1) a capability to quantitatively represent the key SA concepts such as situations, events, and the pilot's mental model; 2) a mechanism to reflect both diagnostic and inferential reasoning; and 3) an ability to deal with various levels and types of uncertainties, since most real-world systems of any complexity involve uncertainty. Russell \& Norvig [14] cite three principal reasons for this uncertainty:

- Theoretical ignorance: All models of physical systems are necessarily approximations

- Laziness: Truly exceptionless rules require numerous antecedents and consequents (cf. 'Frame Problem' [16]) and are computationally intractable
- Practical ignorance: Even if all rules are known, we do not always have time to measure all properties of the objects that must be reasoned over

The principal advantages of belief networks over other uncertain reasoning methods are:

- Its probability estimates are guaranteed to be consistent with probability theory

- It is computationally tractable. Its efficiency stems principally from exploitation of conditional independence relationships over the domain

- The structure of a BN captures the cause-effect relationships that exist among variables of the domain. The ease of causal interpretation in $\mathrm{BN}$ models makes them easier to construct [17]

- The BN formalism supports many reasoning modes: causal reasoning, diagnostic reasoning, mixed causal and diagnostic reasoning, and intercausal reasoning (multiple causes for a given effect). No other uncertain reasoning formalism supports this range of reasoning modes [15]

Belief networks provide the capability and flexibility of modeling SA with its full richness. They also provide a comprehensible picture of the SA problem by indicating dependent relationships among variables, at both high-levels (symbolic) and low-levels (numeric). This provides a clearer view of how each individual piece of evidence affects the high-level situation characterization. They allow the incremental addition of evidence at any network node as it arrives, thus allowing for real-time SA update. Finally, BNs enable a designer to partition a large knowledge base into small clusters, and then specify probabilistic relationships among variables in each cluster (and between neighboring clusters). This approach facilitates construction of large, robust knowledge bases without explicitly specifying the relationships between all possible combinations of variables.

The belief network model shown in Figure 4 was developed to model the pilot's assessment of the protected zone conflict hazard posed by an intruder aircraft (and visible to the pilot via a hypothetical cockpit display of traffic information). As shown, the conflict hazard (which may be none, low, medium, or high) is expressed as a function of four quantities:

1) The range rate (i.e., the rate of change of distance between the intruder and self), which may be positive large, positive small, zero, negative small, or negative large.

2) The aspect angle, which may be zero, low, medium, or high. The aspect angle is the angle between the bearing line to the intruder and the intruder's velocity vector (between $-180^{\circ}$ and $\left.180^{\circ}\right)$, and it is defined such that when aspect is 
zero, the intruder is heading directly away from ownship. When the absolute value of the aspect angle is $180^{\circ}$, the intruder is heading directly towards ownship.

3) The intruder's maneuvering actions in the lateral plane (turning towards self, turning away from self, or not maneuvering).

4) The predicted point of closest approach, which may lie inside or outside the protected zone of ownship. In turn, this depends on where the point of closest approach is in terms of altitude and range.

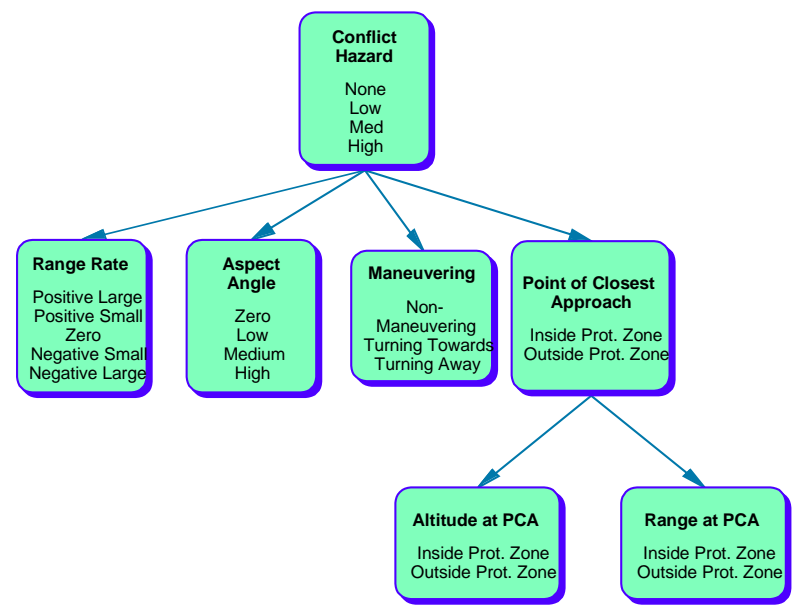

Figure 4: Belief Network Model of Protected Zone Conflict Hazard

A simple conflict model was developed to declare a conflict if the predicted point of closest approach of an intruder lies within the protected zone. Figure 5 illustrates the conflict model, which shows the intruder's position and velocity in a frame of reference fixed to the ownship.

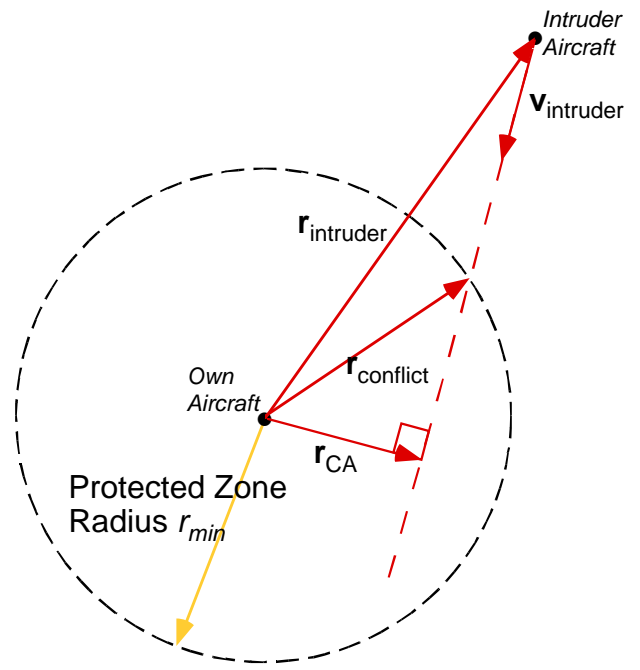

Figure 5: Protected Zone Conflict Model
In terms of the intruder's position $\mathbf{r}_{\text {intruder }}$ and velocity vector $\mathbf{v}_{\text {intruder }}$, the point of closest approach $\mathbf{r}_{C A}$ may be expressed as (assuming constant closure rate):

$$
\mathbf{r}_{\mathrm{CA}}=\mathbf{r}_{\text {intruder }}+\mathrm{t}_{\mathrm{CA}} \mathbf{v}_{\text {intruder }}
$$

where $t_{C A}$ is the time to closest approach. This leads to an equation for the time to closest approach:

$$
t_{C A}=\frac{-1}{V^{2}}\left(\mathbf{r}_{\text {intruder }} \cdot \mathbf{v}_{\text {intruder }}\right) ; V=\left\|\mathbf{v}_{\text {intruder }}\right\|
$$

This expression can be used to compute $\mathbf{r}_{C A}$. Since the range at conflict is known (the radius of the protected zone, $\left.r_{\text {min }}\right)$, another equation can be developed to estimate the intruder's position at the instant of conflict, $\mathbf{r}_{\text {conflict }}$. A conflict is declared if $\mathbf{r}_{C A}$ lies within the protected zone, both in altitude and range.

\section{Implementation of Information Processor using Modern Estimation Technology}

The Information Processor was modeled using two interconnected sub-models: a continuous state estimator and a discrete event detector, emulating a pilot's continuous state estimation and event detection (monitoring) functions. Notice that the latter function also depends upon the events anticipated by the situation assessor.

In the system development, the state estimator was modeled using a Kalman filter [18], with its system model controlled by the detected events as shown in Figure 6. The use of a Kalman filter to model human continuous information processing was first established in the Optimal Control Model (OCM) by Kleinman \& Baron [19]. It has been validated against experimental data in a series of human-machine tasks (e.g. flight-path control), and has been widely accepted as a good representation of human continuous state information behavior. The Kalman filter developed here models the pilot's assessment of an intruder's trajectory with respect to ownship.

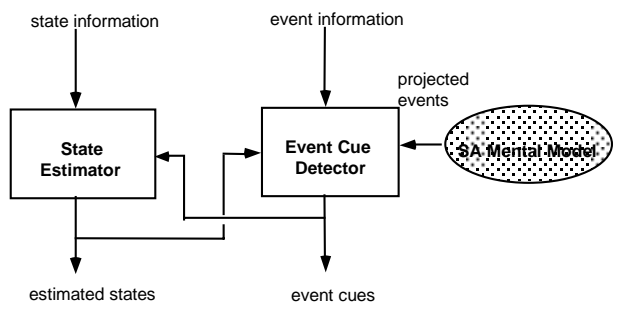

Figure 6: Information Processor Model Using Modern Estimation Techniques

Attention-Sharing Model

For the purposes of implementing a model of human information processing of intruder aircraft information (as seen on a hypothetical CDTI), a means 
of simulating the pilot's attention distribution over multiple potential air traffic hazards is required. It seems reasonable to expect that a pilot would have degraded SA of those intruders receiving less attention (for example, on the basis of their conflict hazard - less threatening intruders would likely receive less attention). Attention distribution was modeled as an effective increase in nominal noise-to-signal ratio on the simulated display [20], via an equation of the form:

$$
\mathrm{P}_{\mathrm{i}}=\mathrm{P}_{0} \frac{1}{f_{t}} \cdot \frac{1}{f_{s}} \cdot \frac{1}{f_{i}}
$$

where $f_{t}$ is the fraction of attention devoted to a control task $i$ as a whole, $f_{s}$ is the attention fraction devoted to subtask $s, f_{i}$ is the attention fraction devoted to the $i^{\text {th }}$ display in subtask $s$, and $P_{0}$ is the nominal noise/signal ratio associated with full attention to the display (typically set to $10 \%$ of signal magnitude).

It was assumed that all of the attention is devoted to observing the cockpit display of traffic information, and that the pilot's attention was divided over multiple air traffic hazards (if any) on the basis of a conflict hazard derived from the belief network described earlier. The BN hazard model provided us with a scalar parameter between 0 and 1 , where 0 represented no threat, and 1 represented high threat (with a network instantiated for each threat visible to the agent).

The total hazard $H$ posed by all visible intruder aircraft was defined as

$$
H=\sum_{i=1}^{n} h_{i}
$$

where $h_{i}$ is the perceived hazard (between 0 and 1) of intruder $i$ and $n$ is the number of detected intruder aircraft. The attention fraction $f_{i}$ on monitoring intruder $i$ was then defined as:

$$
f_{i}=\frac{h_{i}}{H} f_{s} ; \quad 0<f_{i} \leq 1 \text { and } \sum_{i} f_{i}=1
$$

where $f_{s}$ is the fraction of total attention for monitoring air traffic (defined as 1.0). When multiple air traffic hazards are present, the pilot agent's attention is distributed over them in accordance to their perceived conflict threat. It will be shown in the system demonstration that this approach produces intuitively sensible results in a set of simulation trials.

\section{Implementation of Metrics of Pilot Situation Awareness}

A principal goal of this work was to develop a methodology for quantitatively predicting pilot SA as a function of free flight rules and configuration in a multiagent flight environment. Accordingly, a means of extracting quantitative SA/DM performance measures from the pilot agent was required.
The pilot agent architecture provides internal estimates of pilot IP, SA, and DM activities and a timeline of these activities. As a result, a dual metric computation scheme as illustrated in Figure 7 can be employed. For each simulation, two agent models for each vehicle were created:

1) A reference pilot agent who receives perfect information from the simulation but does not control the aircraft.

2) An acting pilot agent that receives the information processed by simulated onboard subsystems (which may contain error) such as flight instruments, datalink, etc., and controls the aircraft simulation.

The reference pilot agent generates ideal IP, SA, and DM activities that are not affected by the onboard subsystems, while the acting pilot agent produces the IP, SA, and DM activities that reflect the limitations imposed by the onboard subsystems and human cognitive capabilities. Comparing the reference and actual IP, SA, DM activities provides a means of measuring the disparity between the ideal activities and the pilot activities, broken down by IP, SA, and DM behaviors. This disparity, in turn, provides a direct metric reflecting how far the active pilot is from the ideal reference pilot, in a set of objective pilotreferenced dimensions characterizing appropriate pilot behavior under free flight.

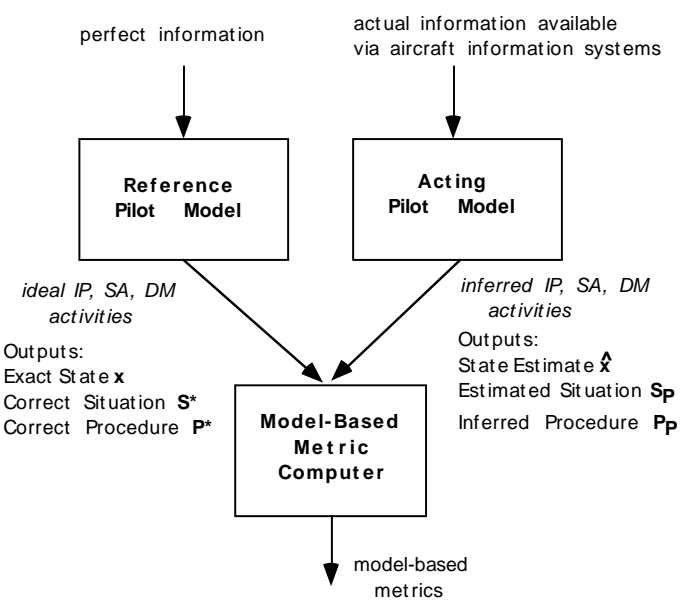

Figure 7: Pilot Agent SA/Performance Metric Generator

A metric of information processing accuracy was obtained by comparing the exact state of an intruder aircraft with that estimated by the pilot agent's Kalman filter tracking the intruder's motion. A measure of information disparity was defined by comparing the exact state $\mathbf{x}$ with the state estimate $\hat{\mathbf{x}}$ :

$$
\mathrm{ID}=[\mathbf{x}(\mathrm{t})-\hat{\mathbf{x}}(\mathrm{t})]^{\mathrm{T}} \Sigma^{-1} \mathbf{x}(\mathrm{t})[\mathbf{x}(\mathrm{t})-\hat{\mathbf{x}}(\mathrm{t})]
$$

where $\Sigma$ is a square matrix used to weight individual terms in the state vector and normalize the result to lie 
between 0 and 1 . The state estimate $\hat{\mathbf{x}}$ feeds the acting pilot model's BN that is tracking the intruder, while the actual state $\mathbf{x}$ feeds a "shadow" BN in the reference pilot model, which computes the true conflict hazard to the ownship posed by the intruder.

A normalized Euclidean distance between the belief values in the acting pilot and reference BNs was then computed, to obtain an overall measure of belief disparity (scaled between 0 and 1 , where 0 represents no error and 1 represents the maximum possible error). When the acting $\mathrm{BN}$ and the reference $\mathrm{BN}$ have the same topology (i.e., the same number of nodes and identical node definitions), the total belief disparity $B D$ is defined as:

$$
B D=\frac{1}{n} \sum_{i=1}^{n} d_{i} ; \quad 0 \leq d_{i} \leq 1
$$

where $n$ is the number of nodes in the belief network, and $d_{i}$ is the disparity at the $i^{\text {th }}$ node. The node disparity is computed as follows. At a given node $j$, assume that there are $k$ mutually exclusive hypotheses for the node's value (e.g., low, medium, high, etc.). The belief vector $\mathbf{b}_{j}$ at that node can then be expressed as

$$
\mathbf{b}_{j}=\left[\begin{array}{llll}
x_{1} & x_{2} \ldots & x_{k}
\end{array}\right] ; \quad \sum_{r=1}^{k} x_{r}=1 \text { and } 0 \leq x_{r} \leq 1
$$

where the $x_{r}$ are the belief values in each of the hypotheses. The node disparity $d_{i}$ is defined as

$$
d_{i}=\frac{\left\|\mathbf{b}_{j_{\text {reference }}}-\mathbf{b}_{j_{\text {actual }}}\right\|}{\sqrt{k}}
$$

The total situational disparity may then be expressed as a weighted average of the terms $B D$ and ID. Preliminary evaluation of these metrics over a range of free flight scenarios discussed in the next section. While these metrics provide an overall measure of SA disparity and support the model feasibility demonstration, they have a number of limitations that will be addressed in the future. In particular, the metrics weight all elements of the belief network disparity equally. This could be remedied easily by introducing a weighting term into equation (7):

$$
B D=\frac{1}{n} \sum_{i=1}^{n} k_{i} d_{i}
$$

where the $k_{i}$ enables weighting of the individual node disparities on the basis of some measure of each node's relevance to overall piloting operations.

\section{SIMULATION RESULTS}

A set of simulation results are now presented to demonstrate the performance of the agent model. First, a two-aircraft scenario is provided to illustrate the effects of sensor accuracy on pilot agent situation awareness metrics. Next, the effects of pilot attention distribution in a three aircraft scenario is investigated. Here, the subject pilot must divide his attention over two potential traffic hazards. Finally, results are presented for a scenario examining the effect of dynamic maneuvering by intruder aircraft on pilot SA.

\section{Effect of Sensor Accuracy}

Figure 8 illustrates the first and second scenarios, used to evaluate the effect of sensor accuracy. Our interest is in the SA of pilot AE1022, shown at the bottom of the diagram. The other aircraft, AC1111, has been positioned such that it will cause a protected zone conflict. The simulated CDTI used by AE1022's information-processing model provides the type of information that might be available from ADSB-B, with an error standard deviation of $7.5 \%$ in the underlying sensor data. Scenario 2 has the same geometry, except that the error in sensor data available to AE1022 has a standard deviation of only $2.5 \%$.

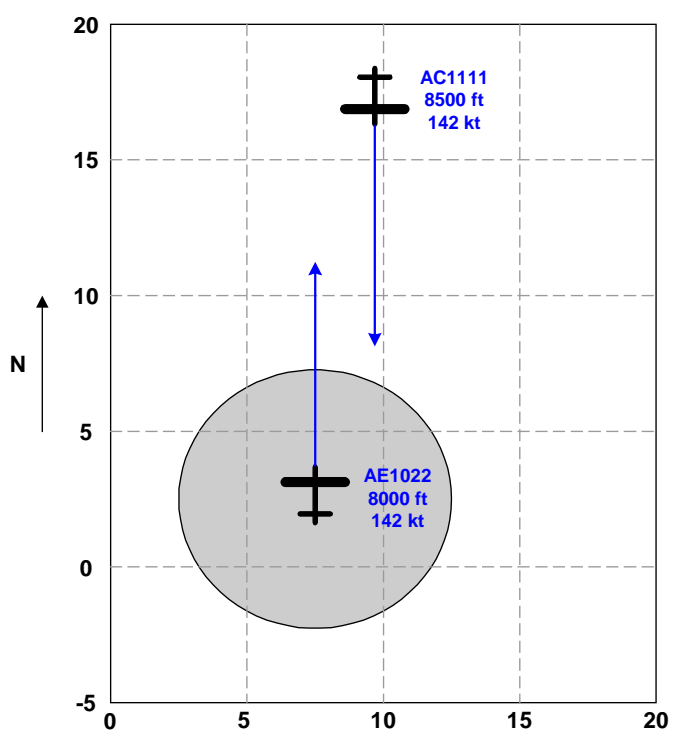

Figure 8: Traffic Scenarios \#1 and \#2 for Evaluation of Sensor Accuracy Effects

Figure 9 shows the perceived and actual conflict hazard perceived by AE1022 in scenario 1 and 2, respectively. It is apparent that reducing the sensor noise reduces the fluctuation and error in the perceived conflict hazard.

Figure 10 compares the SA disparity in Pilot AE1022's awareness of AC1111 across both scenarios. It is quite apparent that the SA disparity is much higher in Scenario 1, in which AE1022's sensor data contained 3 times as much error as in Scenario 2. This finding supports the objective to use model-based metrics as a basis for evaluating the effect of sensor accuracy on pilot SA. Such evaluations would be useful in the development of technologies to enable safe operations under free flight. Specifically, information regarding 
the requirements for information accuracy would aid in the development of sensor hardware and cockpit displays capable of providing adequate situation awareness and appropriate decision support for pilots operating in the free flight environment.

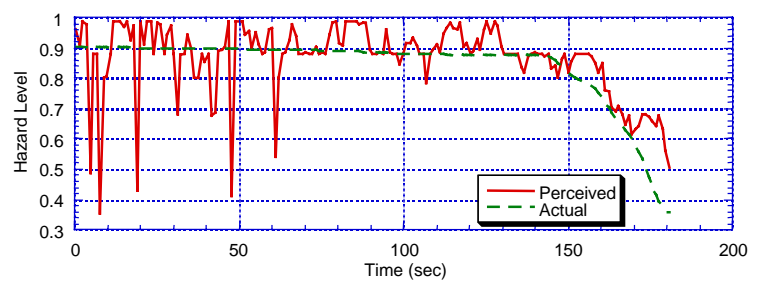

(a) Hazard Perceived by AE1022 in Scenario 1

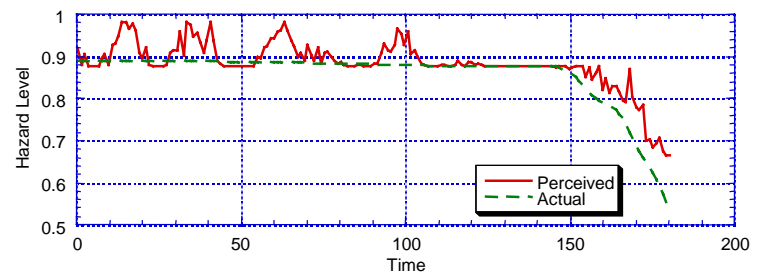

(b) Hazard Perceived by AE1022 in Scenario 2

Figure 9: Perceived Hazard Measures in Scenarios \#1 and \#2

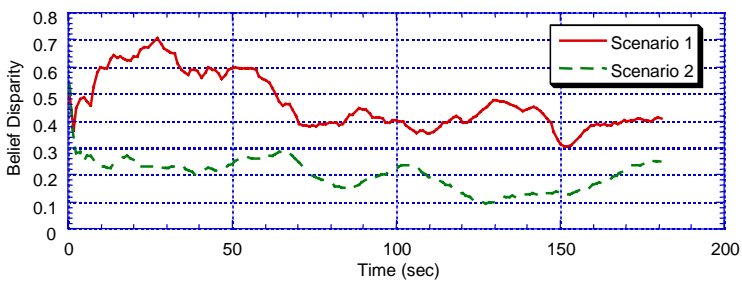

Figure 10: Belief Disparity for Pilot AE1022 in Scenarios \#1 and \#2

\section{Effect of Attention Distribution}

The next scenario, shown in Figure 11, examines the effect of attention-sharing on pilot SA. Sensor noise in monitoring both intruders is set to $2.5 \%$. This scenario is the same as the previous one, except that a third intruder aircraft (UA103) is added. This intruder has been placed such that its flight path will not pose a conflict hazard to AE1022. The aircraft AC1111 has been placed in the same position with the same speed as scenarios 1 and 2 presented above.

Figure 12 shows the hazard perceived by AE1022 from both intruders during the scenario. AC1111 poses a high hazard until approximately $100 \mathrm{sec}$, at which point it begins increasing its distance from AE1022 (although it is still inside AE1022's protected zone). By about $140 \mathrm{sec}$, it exits AE1022's protected zone, causing the perceived hazard to drop even further. The hazard index for UA103 never rises above 0.35 (representing a low hazard), as it never poses any conflict hazard.

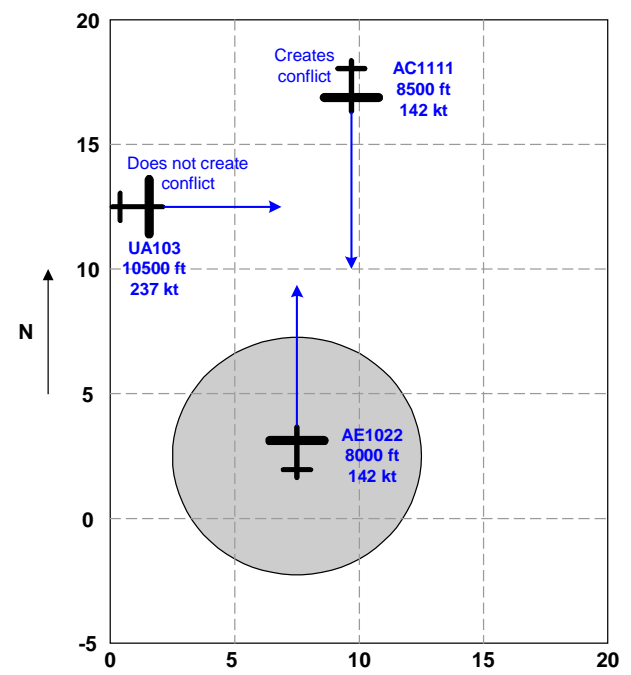

Figure 11: Scenario \#3 for Evaluation of Attention Sharing Effects on SA

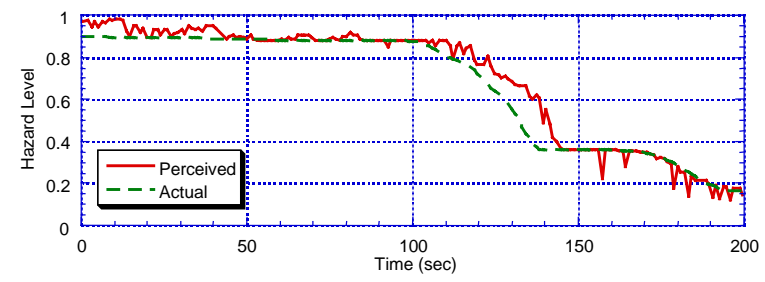

(a) Perceived and Actual Conflict Hazard of AC1111

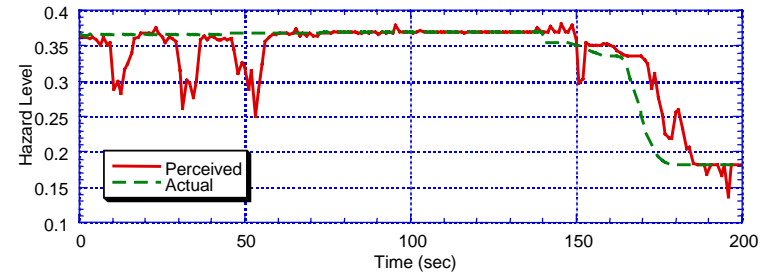

(b) Perceived and Actual Conflict Hazard of UA103

Figure 12: Perceived and Actual Hazard Measures for AE1022 in Scenario \#3

Figure 13 illustrates the effect of pilot AE1022's attention distribution over the two potential hazards during the scenario. Figure 13 a) shows AE1022's belief disparity in monitoring both hazards. Although the sensor error on tracking both intruders is the same, the agent's SA disparity in its awareness of UA103 is generally higher throughout the simulation. This is a direct result of the attention distribution over the two intruders, illustrated in Figure 13b). Until AC1111 exits AE1022's protected zone, AE1022 has about $70 \%$ of its attention on monitoring AC1111, and only $30 \%$ of its attention on UA103. This arises directly from the perceived conflict hazards from both intruders, shown in Figure 12. This result indicates that BN-derived threat measures can be used as a sensible utility function for allocating finite attentional resources during a simulation. Once AC1111 exits the protected zone, the 
attention allocation shifts so that it only receives $50 \%$ of AE1022's attention, as does UA103.

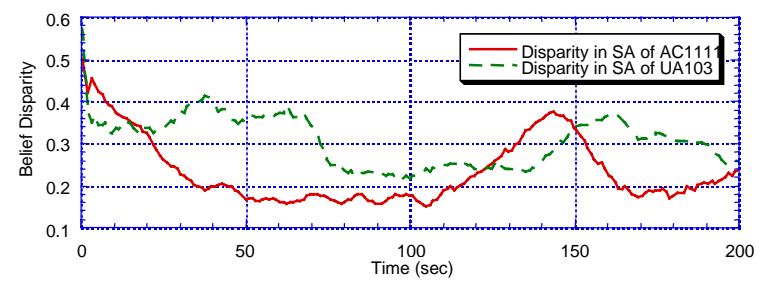

(a) Belief Disparity of Pilot AE1022

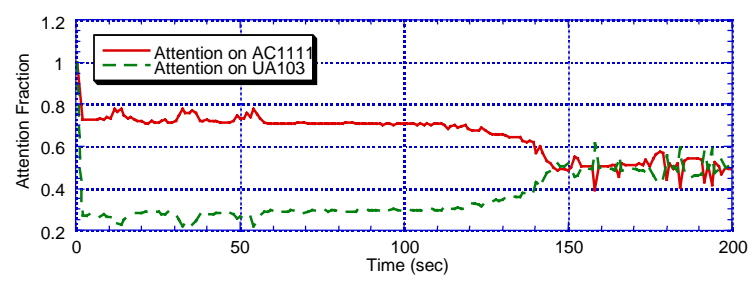

(b) Attention Fraction of Pilot AE1022

Figure 13: Attention Distribution Effects in Scenario \#3

\section{Effect of Dynamic Maneuvering}

The final scenario, shown in Figure 14, examines the effect of dynamic maneuvering by an intruder aircraft on pilot SA. Sensor noise in monitoring both intruders is set to $2.5 \%$. This scenario is the same as the previous one, except that both intruders (AC1111 and UA103) initiate flight path changes that change their conflict hazard to AC1111. AC1111 begins climbing at 30 seconds to prevent the conflict with AE1022. Then, at 60 seconds, UA103 begins descending in such a way as to create a conflict with AE1022.

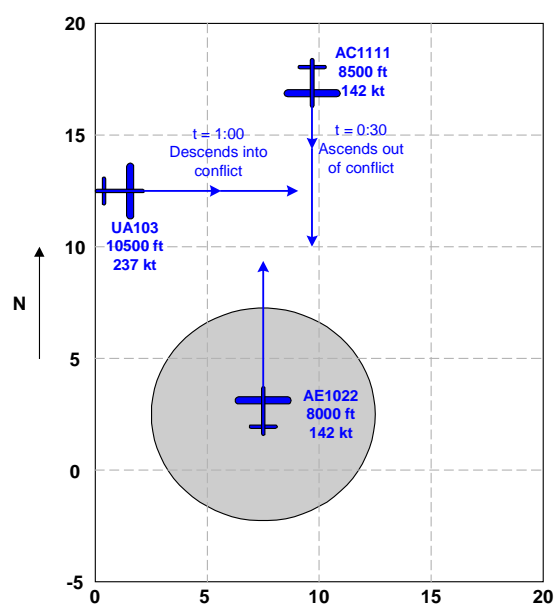

Figure 14: Traffic Scenario \#4 for Evaluation of Maneuvering Effects

Figure 15 shows the hazard perceived by AE1022 from both intruders during the scenario. AC1111 poses a high hazard until approximately 45 seconds into the simulation, at which point its climbing maneuver causes it to no longer pose a conflict hazard with AE1022.
UA103 does not pose a significant hazard to AE1022 until approximately 70 seconds into the simulation, at which time its descent causes its projected flight path to intersect AE1022's protected zone. So, the perceived conflict potential of an intruder does change dynamically in response to maneuvering by the intruder.

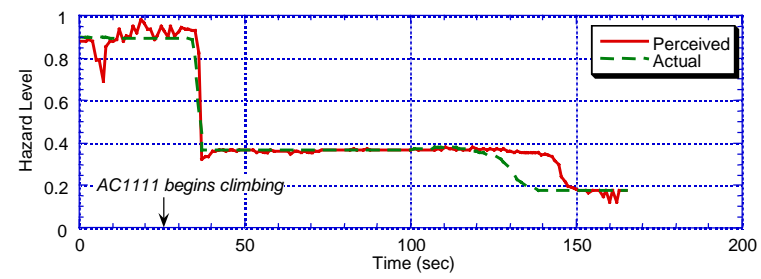

(a) Perceived and Actual Conflict Hazard of AC1111

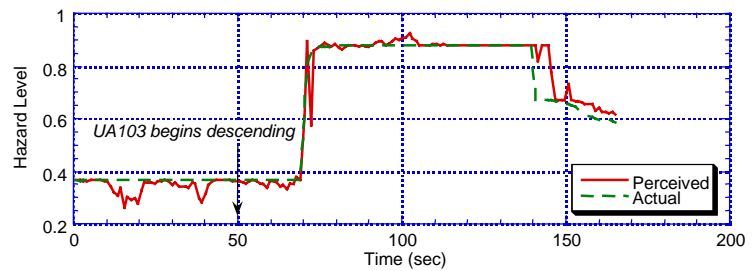

(b) Perceived and Actual Conflict Hazard of UA103

Figure 15: Hazards to AE1022 in Scenario \#4

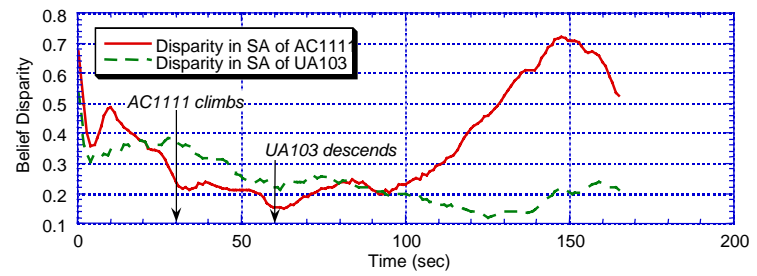

(a) Belief Disparity of Pilot AE1022

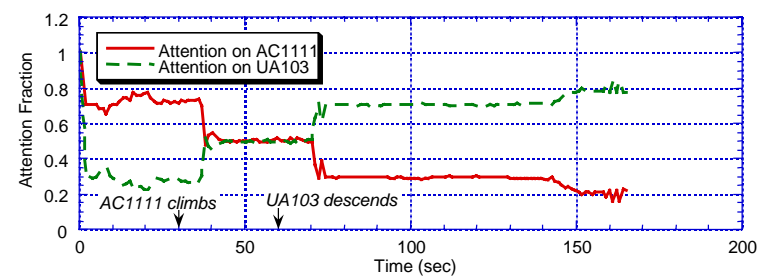

(b) Attention Fraction of Pilot AE1022

Figure 16: Attention Distribution Effects in Scenario \#4

Figure 16 illustrates the effect of pilot AE1022's attention distribution over the two potential hazards, and how the attention distribution is affected by the flight path changes initiated by the two intruders. Initially, most of the pilot's attention is focused on AC1111 since it poses a higher conflict hazard. After AC1111 begins climbing to prevent the conflict, AE1022's attention is divided equally between the two intruders, until approximately 70 seconds into the simulation. At this point, UA103's conflict hazard rises (once its projected flight path intersects AE1022's protected zone). The attention distribution of AE1022 
then shifts to pay more attention to UA103, since it poses a higher conflict hazard. AE1022's drop in attention fraction on monitoring AC1111 correlates with the rise in belief disparity seen in Figure 16 a).

\section{CONCLUSIONS}

The approach to pilot behavioral modeling via representation of information processing, situation assessment, and decision-making presented here allows for the identification of specific errors within this information chain, and lays the groundwork for identifying pilot behavioral errors as they occur. This model-based approach enables the characterization of the pilot's internal awareness of the overall air traffic situation explicitly. This approach, which constructs both an acting pilot model and a reference pilot model, clearly distinguishes between what the pilot does know and what he should know, while relying on a shared mental model of the air traffic environment.

The quantitative SA error metrics employed provide an overall indication of error level, although they do not provide diagnostic information as to error type or cause. However, the modeling approach is general enough that adding these features would be a straightforward extension of the existing model. The simulation results demonstrate the feasibility of a model-based approach for modeling pilot SA and performance in free flight ATM, and lay the foundation for the development of a full-scope tool for pilot performance assessment under free flight

\section{ACKNOWLEDGMENTS}

This work was supported by NASA Ames Research Center, under Contract NAS2-97045. The authors are grateful to Dr. Walt Johnson of NASA Ames for his support during the course of this study.

\section{REFERENCES}

1. FAA. (1995). Advancing Free Flight Through Human Factors. Federal Aviation Administration. (August).

2. RTCA. (1995). Final Report of RTCA Task Force: Free Flight Implementation. RTCA Inc. (October).

3. Garland, D.J., Wise., J.A., Abbott, D.W., et al. (1995). "Free Flight: Human Factors Implications." proceedings of the Human Factors and Ergonomics Society 39th Annual Meeting.

4. Endsley, M.R. (1989). "Situation Awareness Global Assessment Technique (SAGAT)." Proc. of the National Aerospace and Electronics Conference (NAECON). New York, NY. IEEE.

5. Orasanu, J. (1995). "Situation Awareness: Its Role in Flight Crew Decision Making." Proceedings of the 8th International Symposium on Aviation Psychology. Columbus, $\mathrm{OH}$.
6. Scott, W.B. (1996) "New Research Identifies Causes of CFIT," Aviation Week and Space Technology, June 17, pp. 70-71.

7. Nagel, D. (1989). "Human Error in Aviation Operations.” Human Factors in Aviation. E. Wiener and D. Nagel, ed. New York, NY: Academic Press.

8. Zacharias, G., Baron, S., and Muralidharan, R. (1982). "A Supervisory Control Model of the AAA Crew." American Control Conference.

9. Zacharias, G., Miao, A., Illgen, C., Yara, J., and Siouris, G. (1996). "SAMPLE: Situation Awareness Model for Pilot-in-the-Loop Evaluation," First Annual Conference on Situation Awareness in the Tactical Air Environment, Naval Air Warfare Center, Patuxent River, MD.

10. Huettner, C.H. \& Lewis, M.S. (1997). "Aviation Safety Program: Report to Industry 8/13/97”, Available online at http://www.hq.nasa.gov/office/aero/oashttp/curevent/assist.htm.

11. Klein, G. (1989). "Recognition-Primed Decisions." Advances in Man-Machine Systems Research. W.B. Rouse, ed. Greenwich, CT: JAI. 47-92.

12. Smith, C.L. and Sage, A.P. (1991). "Situation Assessment in Command and Control." Information Technology for Command and Control. S.J. Andirole and S.M. Halpin, ed. 449466.

13. Pearl, J. (1988). Probabilistic Reasoning in Intelligent Systems: Networks of Plausible Inference. San Mateo, CA: Morgan Kaufmann.

14. Rouse, W.B. and Morris, N.M. (1985). On Looking into the Black Box: Prospects and Limits in the Search for the Mental Models. Georgia Institute of Technology. DTIC AD-A159080.

15. Russell, P., \& Norvig, P. (1995). Artificial Intelligence: A Modern Approach. Englewood Cliffs, NJ: Prentice Hall.

16. McCarthy, J., \& Hayes, P. J. (1969). "Some Philosophical Problems from the Standpoint of Artificial Intelligence”. In Meltzer, Mitchie, \& Swann (Eds.), Machine Intelligence 4, (pp. 463502). Edinburgh, Scotland: Edinburgh Univ. Press.

17. Henrion, M. (1989). "Some Practical Issues in Constructing Belief Networks". In Kanal, Levitt, \& Lemmer (eds.), Uncertainty in Artificial Intelligence 3. North Holland: Elsevier Science.

18. Kalman, R. (1960). "A New Approach to Linear Filtering and Prediction Problems," ASME Transactions, Journal of Basic Engineering, 82D, pp. 35-50, March.

19. Kleinman, D.L. and Baron, S. (1971). Analytic Evaluation of Display Requirements for Approach to Landing. NASA. CR-1952 (November).

20. Baron, S. and Levison, W.H. (1977). "Display Analysis with the Optimal Control Model of the Human Operator." Human Factors, 19(5). 